\title{
El papel del envejecimiento en el desarrollo de enfermedades cardiovasculares asociadas a patologías renales
}

\author{
Julia Carracedo ${ }^{1,+}$, Guillermo Bodega ${ }^{2,+}$, Rafael Ramírez ${ }^{3,+}$ y Matilde Alique ${ }^{4, *+}$ \\ 1 Departamento de Genética, Fisiología y Microbiología, Facultad de Ciencias Biológicas, Universidad \\ Complutense de Madrid; Instituto de Investigación Sanitaria Hospital 12 de Octubre (imas12); \\ https://orcid.org/0000-0002-8422-812X \\ 2 Departamento de Biomedicina y Biotecnología, Universidad de Alcalá; https://orcid.org/0000-0003-1469- \\ 3242 \\ 3 Departamento de Biología de Sistemas, Universidad de Alcalá (IRYCIS); https://orcid.org/0000-0003-4598- \\ 339X \\ 4 Departamento de Biología de Sistemas, Universidad de Alcalá (IRYCIS); https://orcid.org/0000-0002-7912- \\ 1133 \\ + Estos autores son co-autores \\ † Estos autores comparten autoría co-senior \\ * Autor correspondencia: matilde.alique@uah.es
}

DOI: https://doi.org/10.37536/RIECS.2020.5.1.200

Recibido: 14/04/2020; Aceptado: 21/04/2020; Publicado: 30/05/2020

Resumen: El envejecimiento es considerado un factor de riesgo para el desarrollo de enfermedades cardiovasculares (ECV), y el proceso de senescencia endotelial es el desencadenante de patologías isquémicas y ECV como la arteriosclerosis y la enfermedad renal crónica (ERC). En la senescencia endotelial, las células endoteliales pierden su capacidad de proliferación por el efecto de agentes lesivos como el estrés oxidativo y/o la inflamación generada en el proceso de envejecimiento. Las patologías cardiovasculares, junto al desarrollo de calcificaciones en la pared vascular, presentan una incidencia y prevalencia entre los enfermos con ERC muy superior a la que se observa en la población general. El desarrollo de calcificaciones cardiovasculares es un hallazgo habitual en los enfermos con ERC, sobre todo cuando estos enfermos requieren terapia renal sustitutiva con hemodiálisis (HD). De hecho, entre estos pacientes, las ECV constituyen la principal causa de morbimortalidad, aunque numerosas evidencias indican un carácter plurietiológico para las ECV en los enfermos con ERC. El incremento de estas patologías entre los enfermos en HD sugiere que existen elementos inherentes a la propia HD que actúan como factor patogénico en el desarrollo de las ECV. La caracterización de estos factores para desarrollar elementos de ayuda diagnóstica que permitan identificar enfermos en $\mathrm{HD}$ en riesgo de padecer $\mathrm{ECV}$, y desarrollar terapias que eviten el desarrollo de estas patologías es el objetivo de nuestro grupo de investigación.

Palabras Clave: Enfermedades cardiovasculares, Enfermedad renal crónica, Envejecimiento, Senescencia endotelial.

Abstract: Aging is considered a risk factor for the development of cardiovascular diseases (CVD), and the endothelial senescence process is the trigger for ischemic pathologies and CVD, such as arteriosclerosis. In endothelial senescence, cells lose their proliferation capacity because of damaging agents among them oxidative stress and/or the inflammation generated in the aging process. Endothelial senescence stimulates the development of pathologies associated with age, like CVD and chronic kidney disease (CKD). Cardiovascular pathologies associated with the development of calcifications in the vascular section, present an incidence and prevalence among patients with CKD higher compared with the general population. The development of cardiovascular calcifications is a common finding in patients with CKD, especially when these 
patients are under renal replacement therapy with hemodialysis (HD). Among these patients, CVD are the main cause of morbidity and mortality, although the evidence indicates a multietiological character for CVD in patients with CKD. The increase in these pathologies, particularly between HD patients, may have elements inherent to HD by itself that act as a pathogenic factor in the development of CVD. To date, all studies have focused on the characterization of these factors to develop elements of diagnostic that may detect HD patients at risk of CVD and in the developing of therapies that prevent the development of these pathologies.

Key words: Cardiovascular disease, Chronic kidney disease, Aging, Endothelial senescence.

\section{Introducción}

El envejecimiento de la población es, al menos en parte, el efecto y un gran logro de los avances sociales. Por primera vez en la historia, la mayor parte de la población de nuestro entorno tiene una esperanza de vida igual o superior a los 65 años. En el siglo XXI, alrededor del $7 \%$ de la población mundial tiene 65 años o más, y en los países desarrollados este porcentaje es incluso más alto $(15 \%)$ y continúa creciendo [1]. En la Unión Europea el porcentaje de personas mayores de 65 años superó el 16,1\%, en el año 2000, y se piensa que llegará al 27,5\% en el año 2050; y es previsible que en España se superen estas cifras, estimándose que, para esas fechas, cerca del $35 \%$ de nuestra población superará los 75 años [2,3].

Es incuestionable que este envejecimiento de la población representa un progreso sin parangón en la historia de la humanidad, pero conlleva nuevos desafíos que repercuten de forma particular en el ámbito de la asistencia sanitaria. Envejecer suele llevar implícito cambios morfológicos y fisiológicos que implican una pérdida en las reservas homeostáticas, favoreciendo el desarrollo de las denominadas enfermedades asociadas a envejecimiento (EAE), las cuales, según datos de la Organización Mundial de la Salud (OMS), son la causa del 60\% de las muertes a nivel mundial y desencadenan el $75 \%$ del gasto sanitario público. Las estimaciones para este año, 2020, sin tener presente el efecto de la COVID-19 (enfermedad causada por el SARS-CoV-2), que indiscutiblemente tendrá una repercusión importante al afectar de forma específica a la población de mayor edad, es que estas EAE supondrán el $60 \%$ de la carga global de la enfermedad y serán responsables del $73 \%$ de las muertes en todo el mundo [4,5]. En consecuencia, entender los mecanismos fisiopatológicos que subyacen en las EAE se ha convertido en un área prioritaria de investigación clínica y experimental en salud, en aras de desarrollar nuevas terapias más eficaces y/o encontrar biomarcadores que permitan identificar sujetos en riesgo de desarrollar EAE.

De entre las numerosas patologías que se consideran EAE destacan las enfermedades cardiovasculares (ECV) (cardiopatía isquémica, enfermedades cerebrovasculares, arteriosclerosis...), al haberse convertido en una pandemia que supone una de las primeras causas de morbilidad y mortalidad a nivel mundial. Estas patologías afectan no solo a países industrializados, sino que, en países de ingresos medios-bajos, han superado a las enfermedades infecciosas como primera causa de muerte. De hecho, por su repercusión en la actualidad, las patologías cardiovasculares sobrepasan el ámbito sanitario, para convertirse en un problema social, que promueve, por ejemplo, campañas publicitarias que propugnan modificar nuestros hábitos de vida incluyendo la adaptación de las dietas alimenticias tradicionales para hacerlas cardiosaludables.

La mortalidad de los enfermos con enfermedad renal crónica (ERC) es muy elevada a pesar de las mejoras en el tratamiento renal sustitutivo, y el 50\% de las muertes suele estar asociado a complicaciones cardiovasculares [6-10]. La incidencia de estas patologías es entre 10 y 20 veces superior a la de la población general. En el desarrollo de las ECV, la disfunción y el daño endotelial están presentes en prácticamente la totalidad de los pacientes con ERC, y esto parece ser el inicio de la cascada de eventos que lleva a la ECV.

La inflamación es parte de la fisiopatología de la ERC y se asocia con todos los principales factores de riesgo de disfunción renal modificables. Los marcadores inflamatorios permanecen 
elevados en pacientes con ERC [11]. El principal desencadenante de la respuesta inflamatoria en los pacientes renales es el estrés oxidativo. A su vez, la disfunción endotelial favorece el estrés oxidativo, que potencia la disfunción endotelial [11]. Estudios recientes sugieren que tanto el proceso inflamatorio como el estrés oxidativo en la [11] ERC puede ser consecuencia de un aumento del daño endotelial (de origen multifactorial). Algunos factores relacionados con la ERC, tales como la elevación de toxinas urémicas, la alteración del metabolismo del calcio y del fósforo o el aumento de estrés oxidativo son los causantes de desencadenar y/o acelerar las ECV ocasionadas por otras enfermedades (diabetes, hipertensión, hipercolesterolemia), o bien, por la edad avanzada.

Resulta de vital importancia modular y tratar los factores que ocasionan las ERC y ECV para prevenir el daño en el endotelio vascular, primer estadio y desencadenante de la ECV. Por tanto, el control de la función endotelial puede ser la clave en el desarrollo de terapias que frenen la ECV y sus complicaciones clínicas. Para ello, es muy importante el desarrollo de pruebas diagnósticas que evalúen biomarcadores específicos y modulables en la alteración del endotelio antes de que la ECV se manifieste

\section{Enfermedad cardiovascular y senescencia endotelial}

Thomas Sydenham ya afirmó en 1928 que "un hombre es tan viejo como sus arterias"[12]; un concepto plenamente vigente en la actualidad, con la salvedad de que el término "viejo" probablemente habría que sustituirlo por el concepto de senescencia, que define mejor el envejecimiento biológico. La senescencia se identifica con el deterioro estructural y funcional en órganos y tejidos que conduce a la pérdida de homeostasis y al desarrollo de EAE [13]. Este proceso se produce como una respuesta al potencial daño ocasionado por la acumulación de una gran variedad de agentes inductores de estrés molecular y/o celular que puede observarse asociado a la edad o producirse de forma prematura o acelerada en situaciones de estrés asociadas a diversas patologías [8, 9]. El concepto de senescencia llevaría implícito que las EAE no son causa de daños generalizados que ocurren de forma concurrente en todos los sistemas del organismo; sino que, en sus inicios, suelen afectar a tejidos y órganos aislados hasta generar el proceso sistémico [8, 9, 13-16].

\subsection{Senescencia}

En 1961, Leonard Hayflick y Paul Moorhead [17] descubrieron que fibroblastos humanos en cultivo eran capaces de dividirse un número limitado de veces. Tras este periodo, las células entraban en un estado no proliferativo que denominaron "senescencia celular", sugiriendo que este proceso podría ser la causa subyacente del envejecimiento en humanos.

Las células del organismo, a excepción de las células cancerígenas, poseen una capacidad limitada de proliferación. Una vez que se alcanza este límite, las células entran en un estado de senescencia y, por tanto, cesa su división. Esta entrada en senescencia puede ser programada, como se ha observado en algunos tejidos durante el desarrollo embrionario de ratones [18] y humanos [19]; o también puede desencadenarse como resultado de un daño [20]. Este daño puede ser como consecuencia del proceso de envejecimiento/senectud per se, también conocido como primario o intrínseco, o bien, asociado a patologías que ocasionan un proceso de envejecimiento prematuro como es el caso de las ECV asociadas a patologías renales, o en el caso de otras patologías crónicas.

En el caso del envejecimiento per se, las células que cesan en su división y entran en un estado de senescencia se caracterizan por presentar un fenotipo asociado a células senescentes, conocido en inglés por sus siglas SASP (senescence-associated secretory phenotype) [21, 22]. Sin embargo, las células senescentes originadas por el daño ocasionado por diversas patologías adquieren un fenotipo denominado senescencia prematura o SIPS (stress-induced premature senescence) (Figura 1). En general, las células senescentes se caracterizan por tener un fenotipo secretor, liberando altos niveles de citoquinas pro-inflamatorias, mediadores inmunes, factores de crecimiento y proteasas [21, 23], entre otras sustancias (Tabla I). 


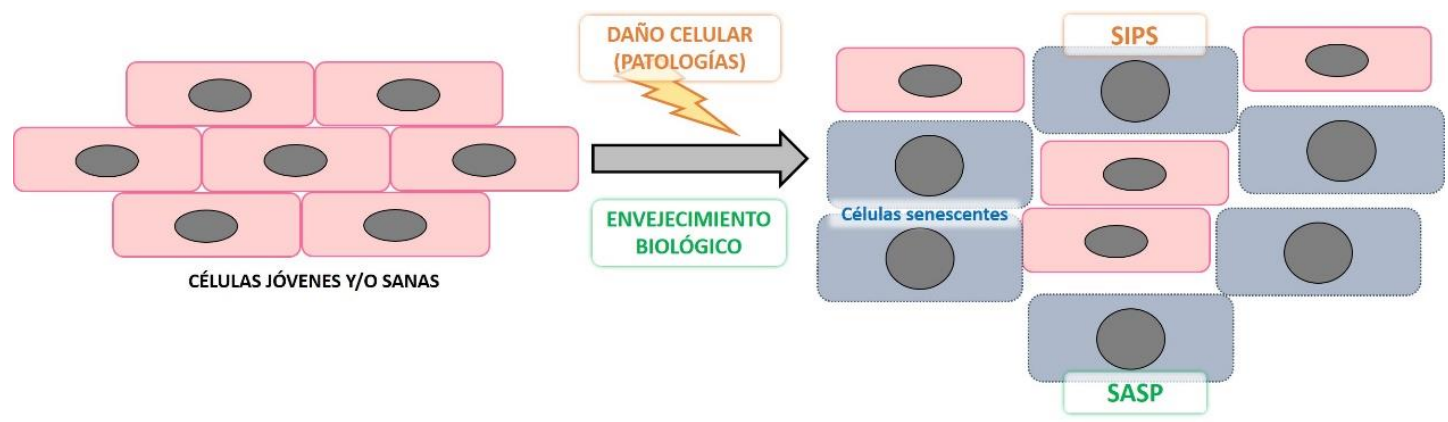

Figura 1 Tipos de senescencia: SASP (senescence-associated secretory phenotype) y SIPS (stress-induced premature senescence)

Tabla I Moléculas implicadas en el proceso de senescencia celular

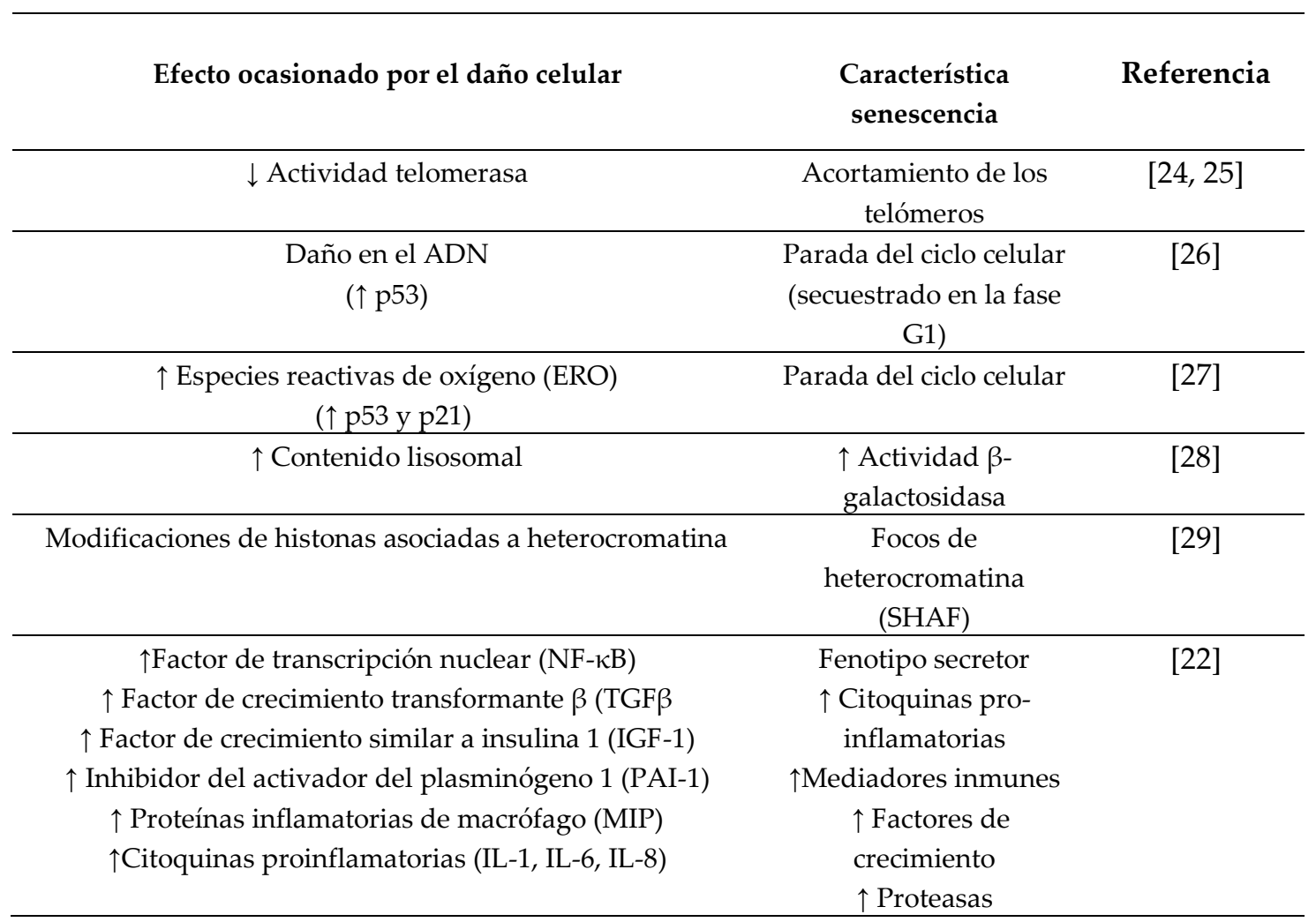

No todas las células que reciben estos estímulos entran en un estado de senescencia, la mayoría de ellas -debido a la magnitud de los daños- son eliminadas directamente mediante el proceso de apoptosis. Los mecanismos exactos que determinan la secuencia que va a seguir una célula (reparación, senescencia o apoptosis) una vez recibe un daño no son bien conocidos, aunque dependen del tipo, duración e intensidad del estímulo [30]. Algunos tipos celulares, al entrar en senescencia, adquieren resistencia a los estímulos proapoptóticos; lo cual explicaría por qué las células senescentes son más estables en cultivo y por qué aumenta el número de células senescentes con la edad [29].

La senescencia no es una patología por sí misma, ya que tiene un papel fisiológico en las células de los tejidos en adultos [20, 31]. En los casos de daño ocasional en un tejido adulto este modelo funciona a la perfección, restaurando por completo el daño del tejido; pero cuando el daño permanece por un tiempo prolongado, este proceso puede verse comprometido haciendo que las células senescentes se acumulen [24]. Las células senescentes se pueden acumular en algunos tejidos adultos 
de forma fisiológica [20]; pero lo hacen con mayor frecuencia en tejidos con patologías crónicas asociadas a la edad como la osteoartritis, fibrosis pulmonar, enfermedad de Alzheimer y en especial en las ECV asociadas a ERC [31]. Esto plantea algunas cuestiones: ¿Promueven las células senescentes enfermedades asociadas a la edad y disfunción en los tejidos? ¿Por qué se acumulan las células senescentes en los tejidos con la edad? Las respuestas a estas preguntas se argumentan a continuación.

Los estímulos que inducen senescencia aumentan con la edad. Con el envejecimiento aparecen unos mayores niveles de estrés oxidativo, se produce un acortamiento de telómeros, y/o se acumulan mutaciones. A su vez disminuye la efectividad con la que se eliminan las células senescentes del tejido, ya por un peor funcionamiento de los mecanismos de competición celular o ya porque el envejecimiento está asociado a un estado de inmunosupresión [32]. Esto ocasiona que las células senescentes se acumulen en el tejido. En el fenotipo senescente SASP se activa una señalización en las células senescentes que provocan la aparición de focos de células senescentes resistentes a la eliminación por parte del sistema inmune, prolongando el estado de senescencia que culmina con la degeneración tisular [31].

Además, las citoquinas y quimioquinas que secretan las células senescentes promueven la fibrosis y la inflamación crónica del tejido por la infiltración de macrófagos y linfocitos, de esta forma se desencadenan enfermedades crónicas asociadas a la edad [33]. Además, las células con SASP secretan metaloproteasas que remodelan la estructura del tejido $y$, junto con las señales proinflamatorias, generan un microambiente que promueve la supervivencia, proliferación y diseminación de las células neoplásicas. Esto explica el aumento de la incidencia del cáncer a partir de ciertas edades [32].

La senescencia favorece el descenso de la capacidad regenerativa de los tejidos propio del envejecimiento. Las células progenitoras terminan entrando en senescencia por un daño o como consecuencia de las secreciones de células senescentes adyacentes. Esto causa una parada en su ciclo y cesa la actividad de los nichos de células madre del tejido. En consecuencia, el tejido pierde su capacidad de regeneración y seguirá acumulando daño, terminando por perder su funcionalidad [32].

Uno de los factores que parecen más claramente implicados en la inducción de daño asociado a la senescencia es el estrés oxidativo, que se produce como consecuencia de un desequilibrio entre la producción de ERO (especies reactivas de oxígeno o en inglés ROS: reactive oxygen species) y la capacidad de los sistemas antioxidantes de la célula de neutralizarlas o de reparar el daño que éstas causan [34]. Esta alteración del estado oxidativo o redox causa daños a los componentes celulares, entre ellos, proteínas, lípidos y ADN [35, 36]. Y puede ocasionar enfermedades de gran importancia clínica como la aterosclerosis [37] y las enfermedades cardiovasculares asociadas a ERC [38]. Además, las ERO son sumamente importantes en el proceso de envejecimiento e incluso en cáncer [39].

La primera teoría sobre la influencia del estrés oxidativo en el envejecimiento la publicó Denham Harman en 1956 [40] y, hasta la fecha, un gran número de investigadores se han centrado en hallar la relación entre estos dos procesos, llegando a varias conclusiones [41]:

1. Los niveles de moléculas dañadas por el estrés oxidativo son mayores en la vejez, pero no se ha podido determinar con exactitud que proteínas [42], lípidos o DNA son los más dañados para comprobar si tienen relación con determinadas vías de señalización que puedan acrecentar los efectos del estrés oxidativo [43].

2. Teóricamente, el empleo de antioxidantes a vertebrados disminuiría el daño oxidativo y aumentaría la vida media. Los experimentos realizados no han proporcionado datos concluyentes, pues una vez se ha producido el daño en la molécula es prácticamente imposible que este revierta mediante antioxidantes; éstos solo protegerán frente a daños nuevos. Sin embargo, estudios en ratones recombinantes con alteraciones en la expresión de enzimas antioxidantes han demostrado que los ratones que aumentan su expresión de enzimas acumulaban menos ERO e iba asociado a un mayor tiempo de vida medio. Por el contrario, 
aquellos animales con una expresión reducida en el patrón de enzimas antioxidantes presentaban un mayor daño oxidativo y disminuía su longevidad [44]. Así, se demostraba que el estrés oxidativo era una de las principales causas del envejecimiento.

La teoría del aumento del estrés y daño oxidativo con la edad concuerda a la perfección con el hecho de que el estrés oxidativo induce senescencia en las células, y por ello existe un mayor número de células senescentes en los tejidos de personas de edad avanzada. Existen varios estudios que corroboran la relación entre las ERO y la senescencia replicativa asociada al envejecimiento [45] y postulan algunas vías que podrían estar regulando la senescencia endotelial inducida por estrés oxidativo [46]. Nuestro grupo ha publicado en los últimos años cómo el patrón en los niveles de estrés oxidativo y de las enzimas antioxidantes de las células endoteliales senescentes se ve ampliamente modulado [47, 48], demostrando así un papel básico en el proceso de envejecimiento endotelial.

\subsection{Senescencia endotelial}

La senescencia endotelial está íntimamente relacionada con las enfermedades asociadas a la edad [49]. Entre las enfermedades más relevantes destacamos las ECV, en especial la arterosclerosis y aquellas patologías cardiovasculares como consecuencia de la ERC en las que tiene lugar una disfunción endotelial [50]. La falta de actividad proliferativa de la célula endotelial senescente explicaría una incapacidad para regenerar el endotelio dañado y/o mantener la actividad angiogénica, terminando por causar un deterioro total de la funcionalidad del vaso que pierde su elasticidad y se vuelve rígido. La importancia en la determinación del fenotipo secretor y, por tanto, de marcadores de senescencia ha abierto nuevas perspectivas en el diagnóstico precoz de ECV y posibilidades de nuevas terapias que puedan frenar o enlentecer la senescencia endotelial.

Prevenir y detener el daño inicial es probablemente la mejor actuación terapéutica para luchar contra las ECV. Desde la aseveración de Sydenham [12] se considera que conocer el estado de salud vascular y prevenir su deterioro es clave para luchar contra esta pandemia. Pero esta tarea no es fácil, la complejidad del sistema vascular y su variabilidad complica enormemente la realización de campañas de prevención de riesgo, como las que se realizan para la detección precoz del cáncer u otras AEA. En este sentido, las campañas preventivas no se dirigen a sujetos en riesgo de desarrollar estas patologías, sino a prevenir en la población general factores como el tabaquismo o la obesidad, asociados ambos a riesgo cardiovascular. Pero ¿cómo prevenir el factor de riesgo que implica la senescencia?

En el año 2002, Minamino y cols. [6] publicaron un estudio que confirmaba una hipótesis manejada desde hace tiempo: la senescencia endotelial es uno de los eventos iniciales en el deterioro vascular que conduce al desarrollo de las ECV asociadas a senescencia. Lejos de la consideración inicial del endotelio como una estructura lineal que recubre la pared vascular para aislar las estructuras internas del torrente sanguíneo, actualmente el endotelio se considera un órgano complejo (hay alrededor de $1 \mathrm{~kg}$ de células endoteliales, 1012 células) [51] que ejerce un amplio rango de actividades funcionales para adaptar en cada momento la respuesta vascular a las diferentes necesidades homeostáticas. En este contexto, el grupo de Minamino [52, 53] demostró que, en patologías como la aterosclerosis, se observaban regiones endoteliales con signos de senescencia prematura en los territorios afectados, sin alterarse el resto de las regiones vasculares. Estos datos abrían unas nuevas perspectivas de abordaje en las ECV ya que, al menos en teoría, era el endotelio vascular el órgano diana para identificar a los sujetos en riesgo de desarrollar estas patologías. Pero afloraba un nuevo problema: no era asumible realizar campañas generalizadas para estudiar el endotelio vascular e identificar sujetos en riesgo. 


\section{Microvesículas endoteliales como marcador de daño endotelial y potencial diana terapéutica}

La práctica totalidad de las células de nuestro organismo producen vesículas extracelulares (VE) como consecuencia de su actividad metabólica, aunque lo hacen en mayor número las células que contactan con líquidos. Las VE son estructuras esféricas rodeadas de una membrana lipídica con un rango de tamaño entre $30 \mathrm{~nm}$ y $1,5 \mu \mathrm{m}$. Fundamentalmente atendiendo a su origen se han subdividido en tres grupos: microvesículas (MV), exosomas y cuerpos apoptóticos. Las dos primeras tienen variadas funciones dependiendo del tipo celular que las origina, aunque su papel más reseñable es la comunicación entre células, siendo capaces de causar cambios estructurales y funcionales en las células diana y, consecuentemente, en los tejidos con los que interaccionan $[1,54]$. Las VE plasmáticas están entre las más abundantes y las más heterogéneas pues derivan de leucocitos, eritrocitos, plaquetas y células endoteliales. La formación y liberación de MV a la circulación sanguínea, desde el punto de vista cualitativo (fenotipo) y cuantitativo (cantidad), depende tanto de la célula de origen, como del estímulo responsable de la liberación $[55,56]$, y de factores externos como pueden ser la edad o el sexo [57].

En situaciones de estrés celular, la producción y liberación al medio extracelular de estas MV se incrementa [58,59]. Este hecho ha llevado a cuantificar el número de MV como un biomarcador que refleje situaciones de estrés y daño celular, dado que algunos subtipos de MV expresan proteínas de superficie procedentes de las células que las originan [60]. Concretamente, un amplio grupo de estudios han demostrado que la cuantificación de MV endoteliales permite identificar a sujetos que presentan daño en el endotelio vascular [59-63]. El hecho de que estas MV circulen en plasma, y que su identificación sea relativamente simple, ha llevado a proponer incorporar su determinación como un método de ayuda al diagnóstico en muchos servicios asistenciales, y su utilidad en el despistaje clínico de sujetos en riesgo de desarrollar ECV parece confirmarse con cada nuevo estudio publicado. Aunque existen discrepancias en la literatura respecto a cómo afecta la senescencia endotelial a la producción de MV desde un punto de vista cuantitativo [64-66], las células endoteliales senescentes producen mayor cantidad de MV que las células jóvenes en modelos experimentales [67]. Además, al menos en el grupo de sujetos ancianos que hemos analizado en nuestros laboratorios, también hemos observado que se produce un incremento en el número de MV endoteliales plasmáticas [67], por lo que aun cuando no podemos confirmar que sean un marcador de daño endotelial asociado a envejecimiento, su número en plasma parece reflejar la edad biológica del endotelio; entendiendo por edad biológica la edad de su estado fisiológico, que no tiene por qué coincidir con su edad cronológica.

Como ya se ha mencionado anteriormente, las células, tanto en condiciones fisiológicas como patológicas, liberan una serie de vesículas que dependiendo de su tamaño, composición y manera de producirse se pueden considerar biomarcadores que reflejan el estado celular. Las células endoteliales producen y liberan al torrente sanguíneo MV denominadas microvesículas endoteliales (MVE), y se ha demostrado que el daño vascular está asociado a un incremento plasmático en MVE en enfermos renales [68]. Cabe destacar que enfermos con ERC tratados con terapias menos agresivas para el endotelio vascular presentan variación en cuanto a su número de MVE [69].

Además de su utilidad como biomarcador de ayuda diagnóstica, numerosos estudios han mostrado que las MV actúan como mecanismo patogénico en el desarrollo de diferentes enfermedades al vehicular mediadores alterados procedentes de las células de origen [60,61]. Al formarse la estructura de la MV, esta engloba proteínas y material genético que estaba presente en el citoplasma de la célula parental. Este material es transportado a la célula de destino modificando el comportamiento de esta última, lo que convierte a estas MV en un sistema de señalización intercelular que modula, entre otros, los mecanismos de daño-reparación celular. Así, por ejemplo, en la homeostasis endotelial, la célula dañada produce MV que activan la maduración de otras células endoteliales inmaduras circulantes (las células progenitoras endoteliales o EPC), que se diferencian para sustituir a la célula dañada manteniendo la integridad endotelial [70]. Pero ante situaciones patológicas, las MV vehiculan señales anómalas que pueden inducir una respuesta celular alterada en la célula del entorno y promover el desarrollo de patologías [60, 61]. 
En el contexto de la senescencia endotelial, diferentes grupos de trabajo [62-67, 70-72], entre ellos nuestro grupo, han comprobado cómo además de diferencias cuantitativas, también se observan diferencias en mediadores de señalización celular vehiculados en las MV producidas por las células endoteliales senescentes. Las células endoteliales senescentes no solo producen más MV, sino que estas llevan diferentes cantidades de microRNA (miRNA) [73] o proteínas [47, 48], y presentan diferencias en muchos de estos mediadores de actividad celular [47, 48]. Estas diferencias parecen jugar un papel en muchas de las deficiencias funcionales descritas en el endotelio senescente, ya que hemos observado, por ejemplo, que a diferencia de lo que ocurre con las MV de células endoteliales jóvenes, las MV de las células endoteliales senescentes no promueven la actividad reparadora y regeneradora endotelial, lo que explicaría, al menos en parte, la disfunción endotelial asociada a senescencia [67, 73, 74]. Recientemente, se ha demostrado que las MV endoteliales también intervienen en la regulación de los mecanismos de estrés oxidativo celular [47, 48], actividad que también se ve afectada por la senescencia. La modulación de la actividad de estas MV, o bien la posibilidad de eliminarlas del plasma de ancianos y sujetos en riesgo por otras situaciones patológicas, identifica a las MV endoteliales senescentes como potenciales dianas terapéuticas en la disfunción endotelial asociada a senescencia [47, 48].

Recientemente se ha relacionado la actividad de las MVE como elementos de señalización intercelular, con el contenido de miRNA que transportan [75]; y se ha demostrado la implicación de miRNA en la aparición de enfermedades, incluidas las asociadas a senescencia como la ECV. Estudios iniciales de nuestro grupo han demostrado que existe un patrón diferencial de miRNA en las MVE derivadas de células endoteliales senescentes comparadas con las MVE derivadas de células jóvenes, independientemente de que esta senescencia sea inducida por actividad replicativa endotelial o por situaciones patológicas como la ERC, [63, 74]. Además, las MVE son muy importantes en procesos de angiogénesis y vasculogénesis [60]. Recientemente, nuestro grupo ha demostrado que el miRNA-126 es un mediador fundamental en la formación de vasos y en la reparación celular en un modelo de senescencia replicativa endotelial [73].

\section{Microvesículas endoteliales senescentes como promotores de la senescencia vascular}

Los datos recogidos en la literatura avalan a la senescencia endotelial como uno de los eslabones iniciales de la cadena de eventos que conducen a senescencia y daño vascular, con el subsecuente desarrollo de ECV [76, 77]. Dado que las MV endoteliales producidas por células senescentes pueden actuar como mecanismo patogénico en la disfunción endotelial, y puesto que está ampliamente demostrado su papel como sistema de señalización intercelular, era lógico pensar que podían participar promoviendo el daño en otras estructuras vasculares además del propio endotelio [78]. En este sentido, en las ECV de anciano y de enfermos con patologías que inducen senescencia vascular prematura, una característica inicial de la enfermedad es el desarrollo de calcificación valvular y en la pared de grandes vasos.

La calcificación de estructuras vasculares es un proceso mal conocido, en el que intervienen estructuras internas como las células musculares lisas de la pared vascular, que sufren un proceso de calcificación que las hace similares a células óseas, lo que impide la adaptabilidad valvular o vascular y favorece el desarrollo de patologías. Se ha demostrado que en el desarrollo de calcificación intervienen proteínas y mediadores de mineralización similares a los que regulan el proceso de osificación [67]. Aún están por determinar estas señales que son las causantes del aumento de la susceptibilidad de la célula vascular en este proceso. En un estudio recientemente publicado se ha demostrado que las MVE senescentes llevan un alto contenido en calcio y otras proteínas promotoras de calcificación vascular [67, 74]; cuando células musculares lisas de la pared vascular son cultivadas en presencia de MVE senescentes modifican su actividad para transformarse en células que van a generar calcificación vascular [67, 74]. Además, la capacidad de las MVE estaba correlacionada con su contenido, de manera que aquellas procedentes de células endoteliales envejecidas contenían una mayor cantidad de proteínas pro-calcificantes y una mayor cantidad de calcio en su interior [67].

Los pacientes con ERC presentan una elevada prevalencia de calcificación vascular, estas calcificaciones aparecen de forma precoz y progresan rápidamente, constituyendo una complicación 
grave de su patología renal, cuya aparición dificulta el tratamiento de la ECV y empeora el pronóstico de la misma $[79,80]$. Con este planteamiento sería de gran interés poder evaluar el papel de las MV de pacientes con ERC y su implicación en el desarrollo de calcificación vascular, para poder desarrollar métodos de diagnóstico precoces para poder tratar la ECV asociada a ERC. En la figura 2 se muestra el papel de las MV en el desarrollo de la ECV asociada a senescencia, tanto a SASP [73] como a SIPS [74].

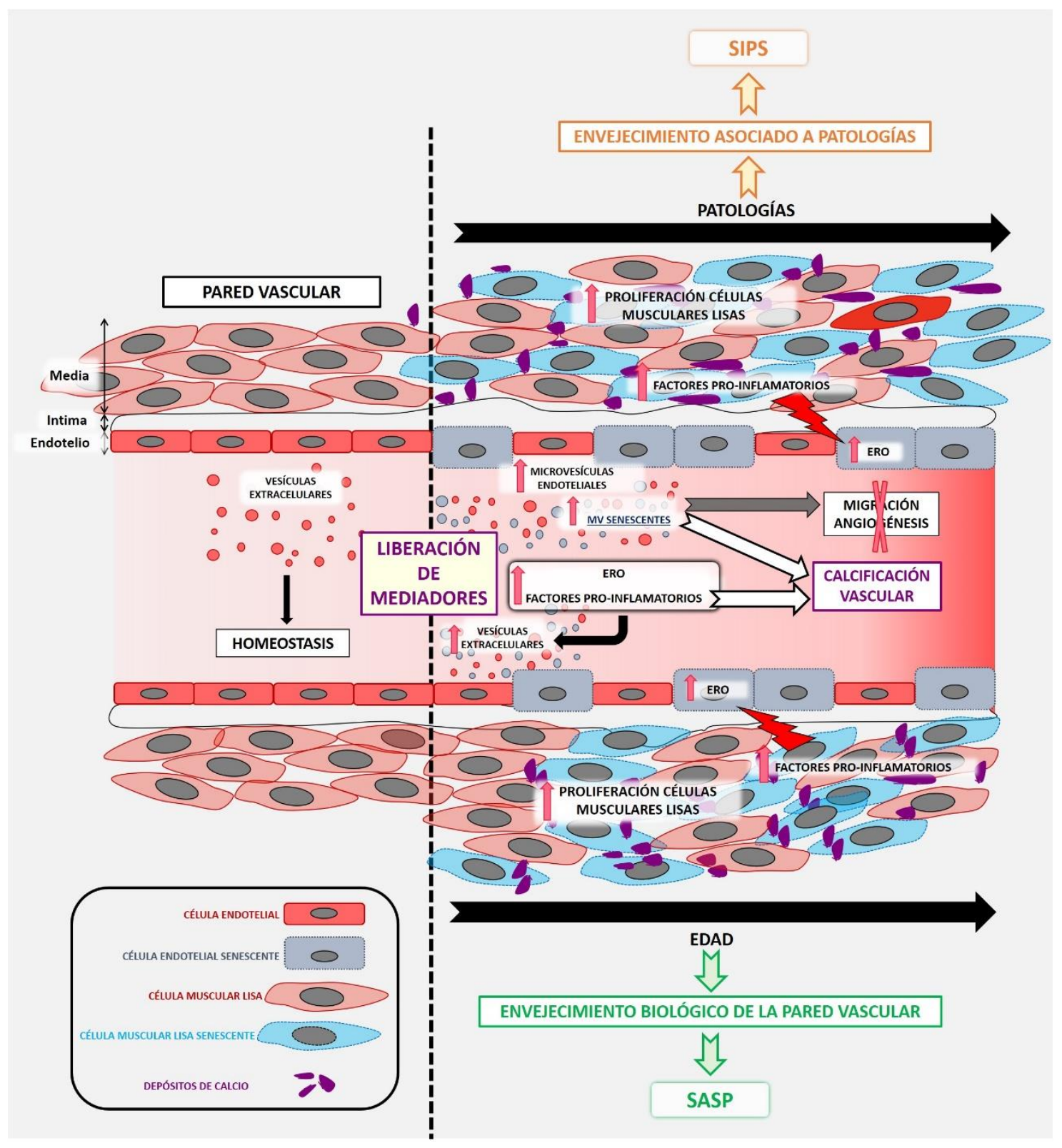

Figura 2 Papel de las MVs en la vasculatura 


\section{Conclusión}

Actualmente, la senescencia celular se considera como la base patogénica por la que numerosos mecanismos etiológicos, fisiológicos (edad) o patológicos inducen daño en el organismo generando patologías. La senescencia endotelial es un elemento inicial en la senescencia vascular y el subsecuente desarrollo de ECV. Los pacientes con ERC desarrollan un proceso de senescencia vascular asociada a la ECV, además de desarrollar calcificaciones cardiovasculares. En este proceso de senescencia, señales vehiculadas en MV de origen endotelial van a actuar promoviendo y expandiendo el proceso de senescencia, lo que hace que estas MV puedan ser utilizadas como biomarcadores y/o dianas terapéuticas en la enfermedad cardiovascular asociada a senescencia en los pacientes con ERC.

Agradecimientos: Este estudio ha sido subvencionado por el Instituto de Salud Carlos III mediante el proyecto "PI17/01029" y “PI19/00240" (Cofinanciado por el Fondo Europeo de Desarrollo Regional "Una manera de hacer Europa"), Santander/UCM, con el proyecto PR41/17-20964, la Sociedad Española de Nefrología (proyecto 2019/2020) y los "Proyectos de Investigación del Programa Propio de la UAH", con los proyectos UAH-GP20184 y CCG2018/BIO-010

Contribución de los autores: "J.C., G.B., R.R., y M.A. han concebido y estructurad la revisión; J.C., G.B., R.R., y M.A realizaron el desarrollo de los diferentes apartados, figuras y tabla; J.C., G.B., R.R., y M.A. escribieron el artículo".

Conflictos de Intereses: los autores no declaran conflicto de intereses.

\section{Abreviaturas}

Las siguientes abreviaturas son usadas en este manuscrito:

COVID-19: Coronavirus disease 2019

EAE: Enfermedades asociadas a envejecimiento

ECV: Enfermedades cardiovasculares

EPC: Células progenitoras endoteliales

ERC: Enfermedad renal crónica

ERO: Especies reactivas de oxígeno

HD: Hemodiálisis

IGF-1: Factor de crecimiento similar a insulina 1

MicroRNA: miRNA

MIP: Proteínas inflamatorias de macrófago

MV: Microvesículas

MVE: Microvesículas endoteliales

NF-kB: Factor de transcripción nuclear

OMS: Organización Mundial de la Salud

PAI-1: Inhibidor del activador del plasminógeno

SARS-CoV-2: Severe acute respiratory syndrome coronavirus 2

SASP: Senescence-associated secretory phenotype

SIPS: Stress-induced premature senescence

TGF $\beta$ : Factor de crecimiento transformante $\beta$

VE: Vesículas extracelulares 


\section{Referencias bibliográficas}

1. Milasan A, Tessandier N, Tan S, Brisson A, Boilard E, Martel C. Extracellular vesicles are present in mouse lymph and their level differs in atherosclerosis. J Extracell Vesicles. 2016;5:31427. PubMed PMID: 27664155. PMCID: PMC5035515. Epub 2016/09/22. eng.

2. WHO, Aging UNIo. Global health and ageing 2011 [Available from: https://www.who.int/ageing/publications/global_health/en/.

3. Coduras Martinez A.. La Sanidad Española en cifras Casal FG, editor2018.

4. Nuño Solinís R. Atención innovadora a las condiciones crónicas: más necesaria que nunca,. Revista de Innovación Sanitaria y Atención Integrada2009.

5. Estrategia de Atención a Pacientes con Enfermedades Crónicas en la Comunidad de Madrid 2013.

6. Minamino T, Miyauchi H, Yoshida T, Ishida Y, Yoshida H, Komuro I. Endothelial cell senescence in human atherosclerosis: role of telomere in endothelial dysfunction. Circulation. 2002 Apr;105(13):1541-4. PubMed PMID: 11927518. eng.

7. Carracedo J, Buendía P, Merino A, Soriano S, Esquivias E, Martín-Malo A, et al. Cellular senescence determines endothelial cell damage induced by uremia. Exp Gerontol. 2013 Aug;48(8):766-73. PubMed PMID: 23624226. eng.

8. Stenvinkel P, Larsson TE. Chronic kidney disease: a clinical model of premature aging. Am J Kidney Dis. 2013 Aug;62(2):339-51. PubMed PMID: 23357108. Epub 2013/01/26. eng.

9. Brownlee M. Advanced protein glycosylation in diabetes and aging. Annu Rev Med. 1995;46:223-34. PubMed PMID: 7598459. eng.

10. Foley RN, Parfrey PS, Sarnak MJ. Clinical epidemiology of cardiovascular disease in chronic renal disease. Am J Kidney Dis. 1998 Nov;32(5 Suppl 3):S112-9. PubMed PMID: 9820470. eng.

11. González Maqueda I, Casanova Rodríguez C, Escobar Cervantes C, Garcia Garcia A, Peraira Moral JR, Prieto Moriche E, Tejero Romero C. Enfermedad cardiovascular y función renal. Mecanismos patogénicos. Cardiovascular Disease and Renal Function. Pathogenetic Mechanisms. Revista Española de Cardiología Suplementos; 2008. p. 10E-21E.

12. Garrison FH. Medical Proverbs, Aphorisms and Epigrams. Bull N Y Acad Med. 1928 Oct;4(10):979-1005. PubMed PMID: 19311626. PMCID: PMC2393983. eng.

13. Childs BG, Durik M, Baker DJ, van Deursen JM. Cellular senescence in aging and age-related disease: from mechanisms to therapy. Nat Med. 2015 Dec;21(12):1424-35. PubMed PMID: 26646499. PMCID: PMC4748967. eng.

14. Sebastiani P, Thyagarajan B, Sun F, Schupf N, Newman AB, Montano M, et al. Biomarker signatures of aging. Aging Cell. 2017 04;16(2):329-38. PubMed PMID: 28058805. PMCID: PMC5334528. Epub 2017/01/06. eng.

15. Belsky DW, Caspi A, Houts R, Cohen HJ, Corcoran DL, Danese A, et al. Quantification of biological aging in young adults. Proc Natl Acad Sci U S A. 2015 Jul;112(30):E4104-10. PubMed PMID: 26150497. PMCID: PMC4522793. Epub 2015/07/06. eng.

16. North BJ, Sinclair DA. The intersection between aging and cardiovascular disease. Circ Res. 2012 Apr;110(8):1097-108. PubMed PMID: 22499900. PMCID: PMC3366686. eng.

17. HAYFLICK L, MOORHEAD PS. The serial cultivation of human diploid cell strains. Exp Cell Res. 1961 Dec;25:585-621. PubMed PMID: 13905658. eng.

18. Storer M, Mas A, Robert-Moreno A, Pecoraro M, Ortells MC, Di Giacomo V, et al. Senescence is a developmental mechanism that contributes to embryonic growth and patterning. Cell. 2013 Nov;155(5):1119-30. PubMed PMID: 24238961. Epub 2013/11/14. eng.

19. Muñoz-Espín D, Cañamero M, Maraver A, Gómez-López G, Contreras J, Murillo-Cuesta S, et al. Programmed cell senescence during mammalian embryonic development. Cell. 2013 Nov;155(5):1104-18. PubMed PMID: 24238962. Epub 2013/11/14. eng.

20. Muñoz-Espín D, Serrano M. Cellular senescence: from physiology to pathology. Nat Rev Mol Cell Biol. 2014 Jul;15(7):482-96. PubMed PMID: 24954210. eng.

21. Coppé JP, Patil CK, Rodier F, Sun Y, Muñoz DP, Goldstein J, et al. Senescence-associated secretory phenotypes reveal cell-nonautonomous functions of oncogenic RAS and the p53 tumor suppressor. PLoS Biol. 2008 Dec;6(12):2853-68. PubMed PMID: 19053174. PMCID: PMC2592359. eng. 
22. Coppe JP, Desprez PY, Krtolica A, Campisi J. The senescence-associated secretory phenotype: the dark side of tumor suppression. Annu Rev Pathol. 2010;5:99-118. PubMed PMID: 20078217. PMCID: PMC4166495. Epub 2010/01/19.

23. Ott C, Jung T, Grune T, Höhn A. SIPS as a model to study age-related changes in proteolysis and aggregate formation. Mech Ageing Dev. 2018 03;170:72-81. PubMed PMID: 28755850. Epub 2017/07/27. eng.

24. Lopez-Otin C, Blasco MA, Partridge L, Serrano M, Kroemer G. The hallmarks of aging. Cell. 2013 Jun 6;153(6):1194-217. PubMed PMID: 23746838. PMCID: PMC3836174. Epub 2013/06/12.

25. Bär C, Bernardes de Jesus B, Serrano R, Tejera A, Ayuso E, Jimenez V, et al. Telomerase expression confers cardioprotection in the adult mouse heart after acute myocardial infarction. Nat Commun. 2014 Dec;5:5863. PubMed PMID: 25519492. PMCID: PMC4871230. Epub 2014/12/18. eng.

26. Chen WY, Wang DH, Yen RC, Luo J, Gu W, Baylin SB. Tumor suppressor HIC1 directly regulates SIRT1 to modulate p53-dependent DNA-damage responses. Cell. 2005 Nov;123(3):437-48. PubMed PMID: 16269335. eng.

27. Chakraborty S, Rasool RU, Kumar S, Nayak D, Rah B, Katoch A, et al. Cristacarpin promotes ER stressmediated ROS generation leading to premature senescence by activation of p21(waf-1). Age (Dordr). 2016 Jun;38(3):62. PubMed PMID: 27246693. PMCID: PMC5005927. Epub 2016/05/31. eng.

28. Debacq-Chainiaux F, Erusalimsky JD, Campisi J, Toussaint O. Protocols to detect senescence-associated beta-galactosidase (SA-betagal) activity, a biomarker of senescent cells in culture and in vivo. Nat Protoc. 2009;4(12):1798-806. PubMed PMID: 20010931. eng.

29. Campisi J, d'Adda di Fagagna F. Cellular senescence: when bad things happen to good cells. Nat Rev Mol Cell Biol. 2007 Sep;8(9):729-40. PubMed PMID: 17667954. eng.

30. Childs BG, Baker DJ, Kirkland JL, Campisi J, van Deursen JM. Senescence and apoptosis: dueling or complementary cell fates? EMBO Rep. 2014 Nov;15(11):1139-53. PubMed PMID: 25312810. PMCID: PMC4253488. Epub 2014/10/13. eng.

31. van Deursen JM. The role of senescent cells in ageing. Nature. 2014 May;509(7501):439-46. PubMed PMID: 24848057. PMCID: PMC4214092. eng.

32. Campisi J, Robert L. Cell senescence: role in aging and age-related diseases. Interdiscip Top Gerontol. 2014;39:45-61. PubMed PMID: 24862014. PMCID: PMC4211612. Epub 2014/05/13. eng.

33. Jurk D, Wilson C, Passos JF, Oakley F, Correia-Melo C, Greaves L, et al. Chronic inflammation induces telomere dysfunction and accelerates ageing in mice. Nat Commun. 2014 Jun;2:4172. PubMed PMID: 24960204. PMCID: PMC4090717. Epub 2014/06/24. eng.

34. Davalli P, Mitic T, Caporali A, Lauriola A, D'Arca D. ROS, Cell Senescence, and Novel Molecular Mechanisms in Aging and Age-Related Diseases. Oxid Med Cell Longev. 2016;2016:3565127. PubMed PMID: 27247702. PMCID: PMC4877482. Epub 2016/05/10. eng.

35. Romá-Mateo C, Seco-Cervera M, Ibáñez-Cabellos JS, Pérez G, Berenguer-Pascual E, Rodríguez LR, et al. Oxidative Stress and the Epigenetics of Cell Senescence: Insights from Progeroid Syndromes. Curr Pharm Des. 2018;24(40):4755-70. PubMed PMID: 30644344. eng.

36. Robinson AR, Yousefzadeh MJ, Rozgaja TA, Wang J, Li X, Tilstra JS, et al. Spontaneous DNA damage to the nuclear genome promotes senescence, redox imbalance and aging. Redox Biol. 2018 07;17:259-73. PubMed PMID: 29747066. PMCID: PMC6006678. Epub 2018/04/13. eng.

37. Rice-Evans CA, Gopinathan V. Oxygen toxicity, free radicals and antioxidants in human disease: biochemical implications in atherosclerosis and the problems of premature neonates. Essays Biochem. 1995;29:39-63. PubMed PMID: 9189713. eng.

38. Daenen K, Andries A, Mekahli D, Van Schepdael A, Jouret F, Bammens B. Oxidative stress in chronic kidney disease. Pediatr Nephrol. 2019 06;34(6):975-91. PubMed PMID: 30105414. Epub 2018/08/13. eng.

39. Kudryavtseva AV, Krasnov GS, Dmitriev AA, Alekseev BY, Kardymon OL, Sadritdinova AF, et al. Mitochondrial dysfunction and oxidative stress in aging and cancer. Oncotarget. 2016 Jul;7(29):44879-905. PubMed PMID: 27270647. PMCID: PMC5216692. eng.

40. HARMAN D. Aging: a theory based on free radical and radiation chemistry. J Gerontol. 1956 Jul;11(3):298-300. PubMed PMID: 13332224. eng.

41. Bokov A, Chaudhuri A, Richardson A. The role of oxidative damage and stress in aging. Mech Ageing Dev. 20042004 Oct-Nov;125(10-11):811-26. PubMed PMID: 15541775. eng.

42. Sohal RS, Mockett RJ, Orr WC. Mechanisms of aging: an appraisal of the oxidative stress hypothesis. Free Radic Biol Med. 2002 Sep;33(5):575-86. PubMed PMID: 12208343. eng. 
43. Sohal RS, Orr WC. The redox stress hypothesis of aging. Free Radic Biol Med. 2012 Feb;52(3):539-55. PubMed PMID: 22080087. PMCID: PMC3267846. Epub 2011/10/24. eng.

44. Salmon AB, Richardson A, Pérez VI. Update on the oxidative stress theory of aging: does oxidative stress play a role in aging or healthy aging? Free Radic Biol Med. 2010 Mar;48(5):642-55. PubMed PMID: 20036736. PMCID: PMC2819595. Epub 2009/12/28. eng.

45. Lawless C, Wang C, Jurk D, Merz A, Zglinicki T, Passos JF. Quantitative assessment of markers for cell senescence. Exp Gerontol. 2010 Oct;45(10):772-8. PubMed PMID: 20117203. ENG.

46. Liu R, Liu H, Ha Y, Tilton RG, Zhang W. Oxidative stress induces endothelial cell senescence via downregulation of Sirt6. Biomed Res Int. 2014;2014:902842. PubMed PMID: 25162034. PMCID: PMC4138737. Epub 2014/08/05. eng.

47. Bodega G, Alique M, Bohórquez L, Ciordia S, Mena MC, Ramírez MR. The Antioxidant Machinery of Young and Senescent Human Umbilical Vein Endothelial Cells and Their Microvesicles. Oxid Med Cell Longev. 2017;2017:7094781. PubMed PMID: 28642812. PMCID: PMC5470024. Epub 2017/05/31. eng.

48. Bodega G, Alique M, Bohórquez L, Morán M, Magro L, Puebla L, et al. Young and Especially Senescent Endothelial Microvesicles Produce NADPH: The Fuel for Their Antioxidant Machinery. Oxid Med Cell Longev. 2018;2018:3183794. PubMed PMID: 29849879. PMCID: PMC5907394. Epub 2018/04/05. eng.

49. Regina C, Panatta E, Candi E, Melino G, Amelio I, Balistreri CR, et al. Vascular ageing and endothelial cell senescence: Molecular mechanisms of physiology and diseases. Mech Ageing Dev. 2016 Oct;159:14-21. PubMed PMID: 27155208. Epub 2016/05/04. eng.

50. Guo J, Lu L, Hua Y, Huang K, Wang I, Huang L, et al. Vasculopathy in the setting of cardiorenal syndrome: roles of protein-bound uremic toxins. Am J Physiol Heart Circ Physiol. 2017 Jul;313(1):H1-H13. PubMed PMID: 28411233. Epub 2017/04/14. eng.

51. Galley HF, Webster NR. Physiology of the endothelium. Br J Anaesth. 2004 Jul;93(1):105-13. PubMed PMID: 15121728. Epub 2004/04/30. eng.

52. Minamino T, Miyauchi H, Yoshida T, Tateno K, Kunieda T, Komuro I. Vascular cell senescence and vascular aging. J Mol Cell Cardiol. 2004 Feb;36(2):175-83. PubMed PMID: 14871544. eng.

53. Minamino T, Komuro I. Vascular cell senescence: contribution to atherosclerosis. Circ Res. 2007 Jan;100(1):15-26. PubMed PMID: 17204661. eng.

54. Emanueli C, Shearn AI, Angelini GD, Sahoo S. Exosomes and exosomal miRNAs in cardiovascular protection and repair. Vascul Pharmacol. 2015 Aug;71:24-30. PubMed PMID: 25869502. PMCID: PMC4838026. Epub 2015/04/11. eng.

55. Xiong J, Miller VM, Li Y, Jayachandran M. Microvesicles at the crossroads between infection and cardiovascular diseases. J Cardiovasc Pharmacol. 2012 Feb;59(2):124-32. PubMed PMID: 21242813. PMCID: PMC3090703. eng.

56. Jayachandran M, Miller VM, Heit JA, Owen WG. Methodology for isolation, identification and characterization of microvesicles in peripheral blood. J Immunol Methods. 2012 Jan;375(1-2):207-14. PubMed PMID: 22075275. PMCID: PMC3253871. Epub 2011/10/29. eng.

57. Tomaniak M, Gąsecka A, Filipiak KJ. Cell-derived microvesicles in cardiovascular diseases and antiplatelet therapy monitoring - A lesson for future trials? Current evidence, recent progresses and perspectives of clinical application. Int J Cardiol. 2017 Jan;226:93-102. PubMed PMID: 27792994. Epub 2016/10/08. eng.

58. Raposo G, Stoorvogel W. Extracellular vesicles: exosomes, microvesicles, and friends. J Cell Biol. 2013 Feb;200(4):373-83. PubMed PMID: 23420871. PMCID: PMC3575529. eng.

59. Vozel D, Uršič B, Krek JL, Štukelj R, Kralj-Iglič V. Applicability of extracellular vesicles in clinical studies. Eur J Clin Invest. 2017 Apr;47(4):305-13. PubMed PMID: 28156006. Epub 2017/02/28. eng.

60. Dignat-George F, Boulanger CM. The many faces of endothelial microparticles. Arterioscler Thromb Vasc Biol. 2011 Jan;31(1):27-33. PubMed PMID: 21160065. eng.

61. Loyer X, Vion AC, Tedgui A, Boulanger CM. Microvesicles as cell-cell messengers in cardiovascular diseases. Circ Res. 2014 Jan;114(2):345-53. PubMed PMID: 24436430. eng.

62. Soriano S, Carmona A, Triviño F, Rodriguez M, Alvarez-Benito M, Martín-Malo A, et al. Endothelial damage and vascular calcification in patients with chronic kidney disease. Am J Physiol Renal Physiol. 2014 Dec;307(11):F1302-11. PubMed PMID: 25339701. Epub 2014/10/22. eng.

63. Carmona A, Agüera ML, Luna-Ruiz C, Buendía P, Calleros L, García-Jerez A, et al. Markers of endothelial damage in patients with chronic kidney disease on hemodialysis. Am J Physiol Renal Physiol. 2017 Apr;312(4):F673-F81. PubMed PMID: 28077371. Epub 2017/01/11. eng. 
64. Forest A, Pautas E, Ray P, Bonnet D, Verny M, Amabile N, et al. Circulating microparticles and procoagulant activity in elderly patients. J Gerontol A Biol Sci Med Sci. 2010 Apr;65(4):414-20. PubMed PMID: 19942591. eng.

65. Markiewicz M, Richard E, Marks N, Ludwicka-Bradley A. Impact of endothelial microparticles on coagulation, inflammation, and angiogenesis in age-related vascular diseases. J Aging Res. 2013;2013:734509. PubMed PMID: 24288612. PMCID: PMC3830876. Epub 2013/10/28. eng.

66. Gustafson CM, Shepherd AJ, Miller VM, Jayachandran M. Age- and sex-specific differences in blood-borne microvesicles from apparently healthy humans. Biol Sex Differ. 2015;6:10. PubMed PMID: 25964851. PMCID: PMC4426551. Epub 2015/05/11. eng.

67. Alique M, Ruíz-Torres MP, Bodega G, Noci MV, Troyano N, Bohórquez L, et al. Microvesicles from the plasma of elderly subjects and from senescent endothelial cells promote vascular calcification. Aging (Albany NY). 2017 03;9(3):778-89. PubMed PMID: 28278131. PMCID: PMC5391231. eng.

68. Cantaluppi V, Gatti S, Medica D, Figliolini F, Bruno S, Deregibus MC, et al. Microvesicles derived from endothelial progenitor cells protect the kidney from ischemia-reperfusion injury by microRNA-dependent reprogramming of resident renal cells. Kidney Int. 2012 Aug;82(4):412-27. PubMed PMID: 22495296. Epub 2012/04/11. eng.

69. Ramirez R, Carracedo J, Merino A, Nogueras S, Alvarez-Lara MA, Rodríguez M, et al. Microinflammation induces endothelial damage in hemodialysis patients: the role of convective transport. Kidney Int. 2007 Jul;72(1):108-13. PubMed PMID: 17429343. Epub 2007/04/11. eng.

70. Leopold JA. Vascular calcification: Mechanisms of vascular smooth muscle cell calcification. Trends Cardiovasc Med. 2015 May;25(4):267-74. PubMed PMID: 25435520. PMCID: PMC4414672. Epub 2014/10/30. eng.

71. Buendía P, Montes de Oca A, Madueño JA, Merino A, Martín-Malo A, Aljama P, et al. Endothelial microparticles mediate inflammation-induced vascular calcification. FASEB J. 2015 Jan;29(1):173-81. PubMed PMID: 25342130. eng.

72. Luna C, Carmona A, Alique M, Carracedo J, Ramirez R. TNF alpha-Damaged-HUVECs Microparticles Modify Endothelial Progenitor Cell Functional Activity. Frontiers in Physiology. 2015 DEC 22 2015;6. PubMed PMID: WOS:000366891800001.

73. Alique M, Bodega G, Giannarelli C, Carracedo J, Ramírez R. MicroRNA-126 regulates Hypoxia-Inducible Factor-1 $\alpha$ which inhibited migration, proliferation, and angiogenesis in replicative endothelial senescence. Sci Rep. 2019 May;9(1):7381. PubMed PMID: 31089163. PMCID: PMC6517399. Epub 2019/05/14. eng.

74. Alique M, Bodega G, Corchete E, García-Menéndez E, de Sequera P, Luque R, et al. Microvesicles from indoxyl sulfate-treated endothelial cells induce vascular calcification in vitro. Computational and Structural Biotechnology Journal: Elservier; 2020.

75. Deregibus MC, Cantaluppi V, Calogero R, Lo Iacono M, Tetta C, Biancone L, et al. Endothelial progenitor cell derived microvesicles activate an angiogenic program in endothelial cells by a horizontal transfer of mRNA. Blood. 2007 Oct;110(7):2440-8. PubMed PMID: 17536014. Epub 2007/05/29. eng.

76. Khan SY, Awad EM, Oszwald A, Mayr M, Yin X, Waltenberger B, et al. Premature senescence of endothelial cells upon chronic exposure to TNF $\alpha$ can be prevented by N-acetyl cysteine and plumericin. Sci Rep. 2017 Jan;7:39501. PubMed PMID: 28045034. PMCID: PMC5206708. Epub 2017/01/03. eng.

77. Carrecedo J R-CR, Alique M, Ramírez-Chamond R. Endothelial cell senescence in the pathogenesis of endothelial dysfunction: Intech; 2018.

78. Carracedo J, Alique M, Ramirez-Carracedo R, Bodega G, Ramirez R. Endothelial Extracellular Vesicles Produced by Senescent Cells: Pathophysiological Role in the Cardiovascular Disease Associated with all Types of Diabetes Mellitus. Curr Vasc Pharmacol. 2018 Aug. PubMed PMID: 30124156. Epub 2018/08/19. eng.

79. Kendrick J, Chonchol M. The role of phosphorus in the development and progression of vascular calcification. Am J Kidney Dis. 2011 Nov;58(5):826-34. PubMed PMID: 21956015. PMCID: PMC3199354. Epub 2011/09/28. eng.

80. Wilson PW, Kauppila LI, O'Donnell CJ, Kiel DP, Hannan M, Polak JM, et al. Abdominal aortic calcific deposits are an important predictor of vascular morbidity and mortality. Circulation. 2001 Mar;103(11):1529-34. PubMed PMID: 11257080. eng. 
(C) 2020 por los autores; Esta obra está sujeta a la licencia de Reconocimiento 4.0 Internacional de Creative Commons. Para ver una copia de esta licencia, visite http://creativecommons.org/licenses/by-nc-nd/4.0/. 